Notre Dame Journal of Formal Logic

Volume 35, Number 1, Winter 1994

\title{
Some Logics Related to von Wright's Logic of Place
}

\author{
RAMON JANSANA
}

\begin{abstract}
In this paper we study some logics related to the logic of place introduced by von Wright and studied by Segerberg. For every $n \geq 1$ we study the logic of the class of frames whose accessibility relation $R$ satisfies the following condition: if $x \neq y$ then there is $j \leq n$ such that $x R^{j} y$. For a fixed $n \geq 1$ the logic is the one axiomatized by $\mathbf{K}+[n] \varphi \rightarrow[n+1] \varphi+\varphi \rightarrow[n]\langle n\rangle \varphi$, which we call Kn.4B, where $[n] \varphi$ is $\varphi \wedge \square \varphi \wedge \ldots \wedge \square^{n} \varphi$. We prove that these logics are canonical and hence complete, and that they have the finite model property, being thus decidable. We also characterize their classes of frames. In the way of studying them we also study the logics $\mathbf{K}+[n] \varphi \rightarrow[n+1] \varphi$, called $\mathbf{K n . 4}$, and $\mathbf{K}+\varphi \rightarrow[n]\langle n\rangle \varphi$, called Kn.B. A translation between these logics and $\mathbf{S 5}$ is also presented, and the relation among them all is established.
\end{abstract}

1 Introduction G.H. von Wright [6] presents the modal logic of place where the box is interpreted intuitively as "everywhere else." In this interpretation we can assume that sentences express propositions that can be true or false of places. Under this assumption a sentence of the form $\square \varphi$ will be true of a place $i$ if the sentence $\varphi$ is true in every other place that can be reached from $i$, and a sentence of the form $\diamond \varphi$ is true of a place $i$ if the sentence $\varphi$ is true in some other place that can be reached from $i$. von Wright also assumed that each place can be reached from every other place. With this interpretation von Wright put forward the normal modal logic with the following axioms:

$$
\begin{aligned}
& \square \varphi \wedge \varphi \rightarrow \square \square \varphi \\
& \varphi \rightarrow \square \vee \varphi,
\end{aligned}
$$

as the one that better codifies the intuitions just described. Von Wright's logic of place is, as was shown by Segerberg [5], the logic of the class of frames whose accessibility relation is the nonidentity (i.e. $x R y$ iff $x \neq y$ ). And its frames are the symmetric

Received March 18, 1992; revised June 21, 1993 
and weakly transitive ones (if $x R y$ and $y R z$ then $x R z$ or $x=z$ ). This last condition is called aliotransitivity by Segerberg.

In this paper we present a joint study of the logic of place and some natural weakenings of it. For every $n \geq 1$ we study the logic of the class of frames whose accessibility relation $R$ satisfies the following condition: if $x \neq y$ then there is $j \leq n$ such that $x R^{j} y$. The frames of this logic are, as we will see, the frames we call $n$ symmetric and weakly $n$-transitive (see Definitions 4.2 and 3.4). In the course of the work we study also the logic whose class of frames is the class of weakly $n$-transitive frames and the logic whose class of frames is the class of $n$-symmetric frames.

Intuitively our logic for the case $n$ can be seen as codifying the logic of "in every other place that can be reached in fewer than $n+1$ steps." In the Kripke frames the steps are represented by the accessibility relation in the following way: indices represent places, and from one place $x$ a place $y$ can be reached directly if $x R y$. And from a place $x$ a place $y$ can be reached in $j$ steps if $x R^{j} y$.

The logics that I call Kn.4 with axiomatization $\mathbf{K}+[n] \varphi \rightarrow[n+1] \varphi$ have been studied for example in Blok and Köhler [1], where their algebraic semantics are presented, and in Rautenberg [4], where some of their main features are expounded in the main text (pp. 164, 173, 324, 327) and in some exercises (Ex. 5, p. 145 and Ex. 2, p. 154). For the sake of completeness we prove canonicity of these logics and characterize their classes of Kripke frames. We do not study their algebraic semantics.

2 Preliminaries The language of propositional (or sentential) modal logic that we assume in the paper has one binary connective, $\rightarrow$, a propositional constant interpreted always as false, $\perp$, and the modal operator, $\square$. In the paper we use standard notation in modal logic, which the reader can find in Hughes and Cresswell [3].

Let us introduce the following notation: $[0] \varphi$ is $\varphi,[n+1] \varphi$ is $\square^{n+1} \varphi \wedge[n] \varphi,\langle 0\rangle \varphi$ is $\varphi$ and $\langle n+1\rangle \varphi$ is $\diamond^{n+1} \varphi \vee\langle n\rangle \varphi$, where $\square^{n} \varphi$ and $\nabla^{n} \varphi$ are defined as follows: $\square^{0} \varphi$ is $\varphi, \square^{n+1} \varphi$ is $\square \square^{n} \varphi, \diamond^{0} \varphi$ is $\varphi$, and $\diamond^{n+1} \varphi$ is $\diamond \diamond^{n} \varphi$.

The logics we will study in this paper are the normal modal logics axiomatized by the axioms (n.4) and (nB) below:

n.4

$$
[n] \varphi \rightarrow[n+1] \varphi,
$$

which for $n=1$ is equivalent to the first axiom of von Wright, for $n=0$ is equivalent to the axiom $\varphi \rightarrow \square \varphi$, and for $n>1$ the relation that holds between the axiom for the case $n$ and the axiom for the case $n-1$ is the same that holds between the case 1 and the case 0 . And,

$$
\text { nB } \varphi \rightarrow[n]\langle n\rangle \varphi,
$$

which for $n=1$ gives the same normal logic as the one given by von Wright's second axiom, for $n=0$ is the tautology $\varphi \rightarrow \varphi$, and for $n>1$, the relation that holds between the axiom for the case $n$ and the axiom for the case $n-1$ is the same that holds between the case 1 and the case 0 .

First of all we will study, once $n$ is fixed, the normal modal logic axiomatized by the axiom $[n] \varphi \rightarrow[n+1] \varphi$. We call this logic Kn.4. In the second place we will study the normal modal logic axiomatized by the axiom $\varphi \rightarrow[n]\langle n\rangle \varphi$, which we call Kn.B. Finally we will study the normal modal logic axiomatized by the axioms $[n] \varphi \rightarrow[n+1] \varphi$ and $\varphi \rightarrow[n]\langle n\rangle \varphi$, which we call Kn.4B. 
3 Kn.4 logics In this section we study the Kn.4 logics. First of all we prove some syntactic lemmas for further use, then we study the Kripke frames for the Kn.4 logics and prove completeness relative to frames. Finally we study the relationship between the Kn.4 logics (for $n>0$ ) and the logic $\mathbf{S 4}$ using a generalization of a translation of Goldblatt in [2].

Lemma 3.1 For every formula $\varphi$, every $n$ and every $m$, the formula $[n][m] \varphi \leftrightarrow$ $[n+m] \varphi$ is a theorem of $\mathbf{K}$.

Proof: By induction on $n$. If $n=0$ the claim is obvious. Assume it holds for $n$. By definition of $[n+1], \mathbf{K} \vdash[n+1][m] \varphi \leftrightarrow \square^{n+1}[m] \varphi \wedge[n][m] \varphi$. But K $\vdash$ $\square^{n+1}[m] \varphi \leftrightarrow \square^{n+1} \square^{m} \varphi \wedge \ldots \wedge \square^{n+1} \square \varphi \wedge \ldots \wedge \square^{n+1} \varphi$. Hence, since $\mathbf{K} \vdash[n][m] \varphi \rightarrow$ $\square^{n+m} \varphi \wedge \ldots \wedge \square^{n+1} \varphi$, we get that $[n+1][m] \varphi \leftrightarrow \square^{n+1} \square^{m} \varphi \wedge[n][m] \varphi$ is a theorem of $\mathbf{K}$. By inductive hypothesis we get that $\mathbf{K} \vdash[n+1][m] \varphi \leftrightarrow \square^{n+1} \square^{m} \varphi \wedge[n+m] \varphi$.

Lemma 3.2 For every modal formula $\varphi$, and every $m$, the formula $[n] \varphi \rightarrow[n+m] \varphi$ is a theorem of Kn.4.

Proof: By induction on $m$. If $m=0$ the claim is obvious. Assume that it holds for $m$. In this case, by Lemma 3.1, Kn.4 $\vdash[n] \varphi \rightarrow[n][m] \varphi$. Therefore, using the axiom of Kn.4, Kn.4 $\vdash[n] \varphi \rightarrow[n+1][m] \varphi$. Again by Lemma 3.1, Kn.4 $\vdash[n] \varphi \rightarrow$ $[n+m+1] \varphi$.

Proposition 3.3 The logic Kn.4 can be axiomatized in each one of the following ways:

(i) $\mathbf{K}+[n] \varphi \leftrightarrow[n+1] \varphi$

(ii) $\mathbf{K}+[n] \varphi \rightarrow \square^{n+1} \varphi$

(iii) $\mathbf{K}+[n] \varphi \rightarrow[n][n] \varphi$ (if $n>0)$

Proof: Clearly, given the definition of $[n]$, the logics (i) and (ii) are the logic Kn.4. We see that (iii) is the logic Kn.4. Using Lemmas 3.2 and 3.1 we have that the formulas $[n] \varphi \rightarrow[n+n] \varphi$ and $[n+n] \varphi \rightarrow[n][n] \varphi$ are theorems of $\mathbf{K n . 4}$, so $[n] \varphi \rightarrow[n][n] \varphi$ is also a Kn.4 theorem. On the other hand, since $[n][n] \varphi \rightarrow \square[n] \varphi$ is a theorem of $\mathbf{K}$, we have that $[n] \varphi \rightarrow[n+1] \varphi$ is a theorem of (iii).

We study now the Kripke frames for Kn.4.

Definition 3.4 We say that a relation $R$ on a set $W$ is a weakly $n$-transitive relation if and only if $\forall a, b \in W$, if $a R^{n+1} b$ then there is $j \leq n$ such that $a R^{j} b\left(R^{0}\right.$ is the identity on $W$ ). Hence, $R$ is weakly 0-transitive iff $\forall a, b \in W$, if $a R b$ then $a=b$.

Proposition 3.5 A Kripke frame $\langle W, R\rangle$ is a Kn.4 frame if and only if $R$ is weakly n-transitive.

Proof: Assume that $\langle W, R\rangle$ is a Kn.4 frame and that $u \in W$. Let us take the set $X=\left\{v \in W: \exists j \leq n u R^{j} v\right\}$. Let $e$ be a valuation in $\langle W, R\rangle$ such that $e(p)=X$. Then $u \in e([n] p)$. Hence $u \in e([n+1] p)$ since $\langle W, R\rangle$ is a Kn.4 frame. Therefore we have that $\forall v \in W\left(u R^{n+1} v \rightarrow v \in e(p)\right)$. From this follows that $R$ is weakly $n$-transitive. On the other hand it is easy to see that the formula $[n] p \rightarrow \square^{n+1} p$ is valid on every frame $\langle W, R\rangle$ where $R$ is weakly $n$-transitive.

We will prove that the logics Kn.4 are complete relative to their classes of frames. To prove it we will prove that for every $n$ the canonical frame of the logic Kn.4 is a Kn.4 frame. A logic with this property is usually called canonical. First of all we need the following lemma. 
Lemma 3.6 For every $n$ and every formula $\varphi_{0}, \ldots, \varphi_{n}$ the formula $\square^{n} \varphi_{0} \wedge \square^{n-1} \varphi_{1} \wedge$ $\ldots \wedge \square \varphi_{n-1} \wedge \varphi_{n} \rightarrow \square^{n+1}\left(\varphi_{0} \vee \ldots \vee \varphi_{n}\right)$ is a theorem of Kn.4.

Proof: It is easy to check that for every $j \leq n$

$$
\mathbf{K} \vdash \square^{n} \varphi_{0} \wedge \square^{n-1} \varphi_{1} \wedge \ldots \wedge \square \varphi_{n-1} \wedge \varphi_{n} \rightarrow \square^{j}\left(\varphi_{0} \vee \ldots \vee \varphi_{n}\right)
$$

Hence,

$$
\mathbf{K} \vdash \square^{n} \varphi_{0} \wedge \square^{n-1} \varphi_{1} \wedge \ldots \wedge \square \varphi_{n-1} \wedge \varphi_{n} \rightarrow[n]\left(\varphi_{0} \vee \ldots \vee \varphi_{n}\right)
$$

Therefore,

$$
\text { Kn.4 } \vdash \square^{n} \varphi_{0} \wedge \square^{n-1} \varphi_{1} \wedge \ldots \wedge \square \varphi_{n-1} \wedge \varphi_{n} \rightarrow \square^{n+1}\left(\varphi_{0} \vee \ldots \vee \varphi_{n}\right),
$$

since

$$
\text { Kn.4 } \vdash[n]\left(\varphi_{0} \vee \ldots \vee \varphi_{n}\right) \rightarrow \square^{n+1}\left(\varphi_{0} \vee \ldots \vee \varphi_{n}\right) .
$$

Proposition 3.7 For every $n$, the logic Kn.4 is canonical.

Proof: Let $\left\langle W_{c}, R_{c}, e_{c}\right\rangle$ be the canonical model for Kn.4. Let us see that $R_{c}$ is weakly $n$-transitive. Suppose that $u, v \in W_{c}$ and that $u R_{c}^{n+1} v$. Assume for a contradiction that for every $j \leq n$, no $u R_{c}^{j} v$. So, $u \neq v$. Let $\varphi_{0}, \ldots, \varphi_{n}$ be formulas such that $\varphi_{n} \in u$ but $\varphi_{n} \notin v, \square \varphi_{n-1} \in u$ but $\varphi_{n-1} \notin v, \ldots$, and $\square^{n} \varphi_{0} \in u$ but $\varphi_{0} \notin v$. Then, since by Lemma 3.6 we have that

$$
\square^{n} \varphi_{0} \wedge \square^{n-1} \varphi_{1} \wedge \ldots \wedge \square \varphi_{n-1} \wedge \varphi_{n} \rightarrow \square^{n+1}\left(\varphi_{0} \vee \ldots \vee \varphi_{n}\right) \in u,
$$

$\square^{n+1}\left(\varphi_{0} \vee \ldots \vee \varphi_{n}\right) \in u$. Therefore, $\left(\varphi_{0} \vee \ldots \vee \varphi_{n}\right) \in v$. But this is absurd since for every $j \leq n, \varphi_{j} \notin v$.

Corollary 3.8 For every $n$ the logic $\mathbf{K n . 4}$ is complete relative to the class of its Kripke frames.

Now we are going to study the relationship between the logics Kn.4 and K4. This relationship is the one stated in Corollary 3.8. We need the following two lemmas that we will also use later.

Lemma 3.9 If $\langle W, R\rangle$ is a weakly $n$-transitive frame then,

$$
\forall k \forall x y \in W\left(x R^{k} y \rightarrow \exists j \leq n x R^{j} y\right) .
$$

Proof: By induction on $k$. If $k=0$ the claim is obvious. Assume it holds for $k$. Assume also that $x R^{k+1} y$. Then let $z$ be such that $x R^{k} z$ and $z R y$. By inductive hypothesis take $j \leq n$ such that $x R^{j} z$. Then $x R^{j+1} y$. If $j<n$, we have what we want. If $j=n$, since that frame is weakly $n$-transitive there is $j^{\prime} \leq n$ such that $x R^{j^{\prime}} y$.

Lemma 3.10 If $\langle W, R\rangle$ is a weakly $n$-transitive frame then it is weakly $k$-transitive for every $k \geq n$.

Proof: By the previous lemma.

Proposition 3.11 For every $n, \mathbf{K n}+\mathbf{1 . 4} \varsubsetneqq$ Kn.4. 
Proof: Take into account the frame $\langle\{0,1, \ldots, n+1\}, R\rangle$ where $R$ is the relation defined by:

$$
i R j \text { iff } i<j \text { or }(i=n+1 \text { and } j=0)
$$

This frame is weakly $n+1$-transitive but it is not weakly $n$-transitive. Therefore the axiom of Kn.4 is not valid in it. Hence this axiom in not a theorem of $\mathbf{K n + 1 . 4}$ since this logic is complete relative to the class of weakly $n+1$-transitive frames. On the other hand it is clear that the axiom of $\mathbf{K n + 1 . 4},[n+1] \varphi \rightarrow[n+2] \varphi$, is a theorem of Kn.4, since every weakly $n$-transitive frame is a weakly $n+1$-transitive frame and Kn.4 is complete relative to the class of weakly $n$-transitive frames.

Proposition 3.12 K1.4 $\varsubsetneqq$ K4.

Proof: Since $\square \varphi \rightarrow \square \square \varphi$ is a theorem of $\mathbf{K 4}$, the axiom of $\mathbf{K 1 . 4},[1] \varphi \rightarrow \square^{2} \varphi$, is a theorem of $\mathbf{K 4}$. On the other hand the frame $\langle\{0,1\},\{\langle 0,1\rangle,\langle 1,0\rangle\}\rangle$ is weakly 1-transitive but is not transitive. Hence the axiom of $\mathbf{K 4}$ is not valid in it.

Corollary 3.13 $\quad \mathrm{K} \varsubsetneqq \ldots \varsubsetneqq \mathrm{Kn} \mathbf{+ 1 . 4} \varsubsetneqq \mathrm{Kn.4} \varsubsetneqq \ldots q \mathrm{~K} \mathbf{1 . 4} \varsubsetneqq \mathrm{K4} \varsubsetneqq \mathrm{KO.4}$.

Proof: By Propositions 3.11 and 3.12 we have all the inclusions from left to right except the last one. To have this it is enough to see that the axiom of K0.4, $\varphi \rightarrow \square \varphi$, is not a theorem of $\mathbf{K 4}$ but that $\square \varphi \rightarrow \square \square \varphi$ is a theorem of K0.4.

Proposition 3.14 In the lattice of normal modal logics,

$$
\mathbf{K}=\inf \{\mathbf{K n} . \mathbf{4}: n \in \omega\} .
$$

Proof: If $\varphi$ is not a theorem of $\mathbf{K}$ then, since $\mathbf{K}$ has the finite frame property, there is a finite frame, say of cardinality $n$, on which $\varphi$ is not valid. But every finite frame of cardinality $n$ is weakly $n+2$-transitive. Hence, $\varphi$ is not a theorem of $\mathbf{K n + 2 . 4}$.

We now study the relationship that holds between the logics Kn.4 and S4. It happens that for $n>0$ the logic Kn.4 is such that $[n]$ has the properties of $\square$ axiomatized in the system $\mathbf{S 4}$, that is: for every modal formula $\varphi$ the formulas $[n] \varphi \rightarrow \varphi$ and $[n] \varphi \rightarrow[n][n] \varphi$ are theorems of Kn.4.

Consider the following well known translation: to every modal formula $\varphi$ associate the modal formula one obtains when substituting systematically $\left(\square \psi^{0} \wedge \psi^{0}\right)$ for the subformulas of $\varphi$ of the form $\square \psi$, where $\psi^{0}$ is the translation of $\psi$. Goldblatt [2] proves that for every model $\langle W, R, v\rangle$, every $a \in W$, and every modal formula $\varphi$,

$$
\langle W, R, v\rangle \models_{a} \varphi^{0} \text { if an only if }\langle W, R \cup I d W, v\rangle \models_{a} \varphi .
$$

From this fact it can be easily concluded that a modal formula is a theorem of $\mathbf{S 4}$ if and only if its translation is a theorem of K1.4. We generalize this fact to every $n \geq 1$ by defining in each case an appropriate translation. To do it we first generalize Goldblatt's lemma.

Given a frame $\langle W, R\rangle$ define the relation $R \mathrm{n}(n>0)$ by

$$
R \mathrm{n}=\operatorname{Id}_{W} \cup R \cup \ldots \cup R^{n},
$$

and consider for each $n \geq 1$ the translation $(n)$ between modal formulas defined by

$$
\begin{gathered}
\perp^{(n)} \text { is } \perp \\
p_{j}^{(n)} \text { is } p_{j} \\
(\varphi \rightarrow \psi)^{(n)} \text { is }\left(\varphi^{(n)} \rightarrow \psi^{(n)}\right) \\
(\square \varphi)^{(n)} \text { is }[n] \varphi^{(n)} .
\end{gathered}
$$

We then have the following lemma. 
Lemma 3.15 For every $n \geq 1$, every model $\langle W, R, v\rangle$, every $a \in W$, and every modal formula $\varphi$,

$$
\langle W, R, v\rangle \models_{a} \varphi^{(n)} \text { if and only if }\langle W, R n, v\rangle \models_{a} \varphi .
$$

Proof: By induction on the construction of $\varphi$. It is obvious that it holds for sentential variables and for $\perp$. It is routine to see that if it holds for $\varphi$ and $\psi$ it holds for $(\varphi \rightarrow \psi)$. We prove the only interesting case. Suppose it holds for $\varphi$ and that $\langle W, R, v\rangle \models_{a}(\square \varphi)^{(n)}$. So, $\langle W, R, v\rangle \models_{a}[n] \varphi^{(n)}$. If $c \in W$ and $a R$ n $c$ there is $j \leq n$ such that $a R^{j} c$. Since $a \in v\left(\square^{j} \varphi^{(n)}\right), c \in v\left(\varphi^{(n)}\right)$. By inductive hypothesis, $\langle W, R \mathrm{n}, v\rangle \models_{c} \varphi$. Hence we conclude that $\langle W, R \mathrm{n}, v\rangle \models_{a} \square \varphi$. To prove the other direction of the biconditional assume that $\langle W, R \mathrm{n}, v\rangle \models_{a} \square \varphi$ and that $\langle W, R, v\rangle \nvdash_{a}$ $(\square \varphi)^{(n)}$. Then there is $m \leq n$ such that $a \notin v\left(\square^{m} \varphi^{(n)}\right)$. Let $b \in W$ be such that $a R^{m} b$ and $b \notin v\left(\varphi^{(n)}\right)$. By inductive hypothesis $\langle W, R \mathrm{n}, v\rangle \nvdash_{b} \varphi$. But, since $a R^{m} b$ and $m \leq n, a R \mathrm{n} b$. Therefore $\langle W, R \mathrm{n}, v\rangle \nvdash_{a} \square \varphi$ contradicts the initial assumption.

Lemma 3.16 For every frame $\langle W, R\rangle, R$ is weakly $n$-transitive $(n>0)$ if and only if $R n$ is transitive and reflexive.

Proof: $(\Rightarrow)$ If $\langle W, R\rangle$ is a frame with $R$ weakly $n$-transitive, since by definition $R \mathrm{n}$ is reflexive we have to show that $R$ is transitive. It is enough to prove by induction on $n$ that

$$
\forall k \leq n \forall a, b, c \in W \forall j \leq n\left(a R^{j} b \wedge b R^{k} c \rightarrow a R \mathrm{n} c\right) .
$$

$(\Leftarrow)$ If $R \mathrm{n}$ is transitive and reflexive, it is trivial to check that $R$ is weakly $n$-transitive.

Proposition 3.17 For every $n \geq 1$ and every modal formula $\varphi, \varphi$ is a theorem of $\mathbf{S 4}$ if and only if $\varphi^{(n)}$ is a theorem of Kn.4.

Proof: $(\Rightarrow)$ If $\varphi^{(n)}$ is not a theorem of $\mathbf{K n . 4}$, let $\langle W, R\rangle$ be a weakly $n$-transitive frame where $\varphi^{(n)}$ is not valid. By Lemma 3.15, $\varphi$ is not valid in the frame $\langle W, R \mathrm{n}\rangle$. But this frame is by Lemma 3.16 transitive and reflexive and hence an $\mathbf{S 4}$ frame. Therefore $\varphi$ is not a theorem of $\mathbf{S 4}$.

$(\Leftarrow)$ If $\varphi$ is not a theorem of $\mathbf{S 4}$, let $\langle W, R\rangle$ be a transitive and reflexive frame where $\varphi$ is not valid; it exists since $\mathbf{S} \mathbf{4}$ is complete relative to the class of these frames. Then, since $R \mathrm{n}$ is $R, \varphi$ is not valid in the frame $\langle W, R \mathrm{n}\rangle$. Hence, by Lemma 3.15, $\varphi^{(n)}$ is not valid in $\langle W, R\rangle$. But this frame is a weakly $n$-transitive frame, and so $\varphi^{(n)}$ is not a theorem of Kn.4.

$4 \mathrm{KnB}$ and Kn.4B logics In this section we study the normal modal logics $\mathrm{KnB}$ and Kn.4B obtained when one respectively adds the axiom $\varphi \rightarrow[n]\langle n\rangle \varphi$ to the systems $\mathbf{K}$ and Kn.4. The reason that explains the names given to these logics is that $\varphi \rightarrow \square \diamond \varphi$ is known as B. First of all we prove some syntactic lemmas. Afterwards we study the Kripke frames of the logics mentioned. And finally we study the relationship between the logics Kn.4B (for $n>0$ ) and S5.

Proposition 4.1 The following logics are the logic Kn.4B:

(i) $\mathbf{K n . 4}+\langle n\rangle \varphi \rightarrow[n]\langle n\rangle \varphi$

(ii) Kn.4 $+[n] \varphi \vee[n] \neg[n] \varphi$. 
Proof: (ii) is equivalent to (i) since in $\mathbf{K}$ the formulas $\langle n\rangle \varphi \rightarrow[n]\langle n\rangle \varphi$ and $[n] \neg \varphi \vee$ $[n] \neg[n] \neg \varphi$ are equivalent. (i) is equivalent to Kn.4B: on one hand, since $\langle n\rangle\langle n\rangle \varphi \rightarrow$ $\langle n\rangle \varphi$ is a theorem of Kn.4; $[n]\langle n\rangle\langle n\rangle \varphi \rightarrow[n]\langle n\rangle \varphi$ is also a theorem of it, and since $\langle n\rangle \varphi \rightarrow[n]\langle n\rangle\langle n\rangle \varphi$ is a theorem of $\mathbf{K n . 4 B},\langle n\rangle \varphi \rightarrow[n]\langle n\rangle \varphi$ is also one. On the other hand $\varphi \rightarrow\langle n\rangle \varphi$ is a theorem of $\mathbf{K}$ and so $\varphi \rightarrow[n]\langle n\rangle \varphi$ is a theorem of (i).

Definition 4.2 We say that a binary relation on $W, R$, is $n$-symmetric if and only if for every $a, b \in W$, if there is $j \leq n$ such that $a R^{j} b$ then there exist $k \leq n$ such that $b R^{k} a$.

Obviously $R$ is $n$-symmetric if and only if the relation $R$ n defined in Section 3 is symmetric and if a relation $R$ is symmetric it is $n$-symmetric.

Proposition 4.3 $\quad p \rightarrow[n]\langle n\rangle p$ is valid in a frame $\langle W, R\rangle$ if and only if $R$ is n-symmetric.

Proof: $(\Rightarrow)$ Assume that $p \rightarrow[n]\langle n\rangle p$ is valid in $\langle W, R\rangle$. Assume that $a, b \in W$, that $\exists j \leq n$ such that $a R^{j} b$ and that $\forall k \leq n$ it is not the case that $b R^{k} a$. Let $e$ be a valuation on $\langle W, R\rangle$ such that $e(p)=\{a\}$. In this case $b \notin e(\langle n\rangle p)$. So let $j \leq n$ be such that $a R^{j} b . j \neq 0$ since on the contrary $b R^{0} a$. Hence $a \notin e([n]\langle n\rangle p)$. Therefore $a \notin e(p)$, and this is not the case.

$(\Leftarrow)$ If $\langle W, R\rangle$ is an $n$-symmetric frame and $e$ is a valuation on it then if $a \in e(p)$ and $a \notin e([n]\langle n\rangle p)$, let $j \leq n$ and $b \in W$ be such that $a R^{j} b$ and $b \notin e(\langle n\rangle p)$. Since $R$ is $n$-symmetric, let $k \leq n$ be such that $b R^{k} a$. Since $a \in e(p), b \in e(\langle n\rangle p)$, and this is absurd.

The last proposition gives us that the Kripke frames for the logic Kn.B are the $n$-symmetric frames and that the Kripke frames for the logic Kn.4B are the weakly $n$-transitive and $n$-symmetric frames. In the next propositions we prove that $\mathbf{K n B}$ and Kn.4B are complete relative to their classes of frames.

Proposition 4.4 For every $n \geq 1$ the logic $\mathbf{K}+\varphi \rightarrow[n]\langle n\rangle \varphi$ is canonical and hence complete relative to the class of its frames.

Proof: Let $\left\langle W_{c}, R_{c}, e_{c}\right\rangle$ be the canonical model for $\mathbf{K}+\varphi \rightarrow[n]\langle n\rangle \varphi$. We see that $R_{c}$ is $n$-symmetric. Suppose that $u, v \in W_{c}$ and $\exists j \leq n$ such that $u R_{c}^{j} v$. Assume for a contradiction that $\forall k \leq n$ it is not the case that $v R_{c}^{k} u$. For every $k \leq n$ let $\varphi_{k}$ be such that $\varphi_{k} \in u$ and $\diamond^{k} \varphi_{k} \notin v$. Then $\varphi_{0} \wedge \ldots \wedge \varphi_{n} \in u$. Hence $[n]\langle n\rangle\left(\varphi_{0} \wedge \ldots \wedge \varphi_{n}\right) \in u$ and $\square^{j}\langle n\rangle\left(\varphi_{0} \wedge \ldots \wedge \varphi_{n}\right) \in u$. Therefore $\langle n\rangle\left(\varphi_{0} \wedge \ldots \wedge \varphi_{n}\right) \in$ $v$. But for every $i \leq n, \diamond^{i}\left(\varphi_{0} \wedge \ldots \wedge \varphi_{n}\right) \rightarrow \diamond^{i} \varphi_{i}$ is a theorem of $\mathbf{K}$. Hence $\langle n\rangle\left(\varphi_{0} \wedge \ldots \wedge \varphi_{n}\right) \rightarrow \diamond^{n} \varphi_{n} \vee \ldots \vee \diamond^{1} \varphi_{1} \vee \varphi_{0}$ is also a theorem of $\mathbf{K}$. We conclude that $\nabla^{n} \varphi_{n} \vee \ldots \vee \nabla^{1} \varphi_{1} \vee \varphi_{0} \in v$. Therefore there is $k \leq n$ such that $\nabla^{k} \varphi_{k} \in v$, and this is absurd. Hence $\left\langle W_{c}, R_{c}\right\rangle$ is a frame for $\mathbf{K}+\varphi \rightarrow[n]\langle n\rangle \varphi$. As a consequence this logic is canonical and complete relative to the class of its frames.

Corollary 4.5 For every $n \geq 1$ the logic Kn.4B is canonical and hence complete relative to the class of all its Kripke frames (the weakly $n$-transitive and $n$-symmetric ones).

Proof: By Propositions 3.7 and 4.4.

Next we will see that the logic of the class of frames that satisfy the condition

$$
\forall x y \in W\left(x \neq y \rightarrow \exists j \leq n x R^{j} y\right)
$$

is precisely the logic Kn.4B. To see it we first prove the following three lemmas. 
Lemma 4.6 In every weakly $n$-transitive and $n$-symmetric frame $\langle W, R\rangle$, it holds that:

$$
\forall k \forall x y \in W\left(x R^{k} y \rightarrow \exists j \leq n y R^{j} x\right) .
$$

Proof: Obvious using Lemma 3.9 and $n$-symmetry.

Lemma 4.7 If $\langle W, R\rangle$ is a generated frame, then it is weakly $n$-transitive and $n$-symmetric if and only if it satisfies condition (*) above.

Proof: $(\Leftarrow)$ From $\forall x y \in W\left(x \neq y \rightarrow \exists j \leq n x R^{j} y\right)$ it follows that $\forall x y \in W \exists j \leq$ $n x R^{j} y$. From this we conclude that the frame is weakly $n$-transitive and $n$-symmetric.

$\Rightarrow$ Assume that $t$ is the generator of the frame. If $x \neq y$ let $k$ and $l$ be such that $t R^{k} x$ and $t R^{l} y$. We have that $t \neq x$ or $t \neq y$. If $t \neq x$, let $j \leq n$ be such that $t R^{j} x$. If $y=t, y R^{j} x$. Hence by $n$-symmetry there is $m \leq n$ such that $y R^{m} x$. On the other hand if $y \neq t$, let $j^{\prime} \leq n$ be such that $t R^{j^{\prime}} y$. By $n$-symmetry let $j^{\prime \prime} \leq n$ be such that $y R^{j^{\prime \prime}} t$. Hence $y R^{j^{\prime \prime}+j} x$. By Lemma 4.6 there is $m \leq n$ such that $x R^{m} y$.

Lemma 4.8 If $\left\langle W_{c}, R_{c}, e_{c}\right\rangle$ is the canonical model for $\mathbf{K n . 4 B}$ and $u \in W_{c}$, the subframe generated by $u$ is weakly $n$-transitive and $n$-symmetric.

Proof: Let $W$ be the domain of the subframe generated by $u$ and $R$ its relation. If $x, y \in W$ and $x R^{n+1} y$ then $x R_{c}^{n+1} y$. Let $j \leq n$ be such that $x R_{c}^{j} y$. Let $x, x_{1}, \ldots, x_{j}, y$ be an $R_{c}$ sequence. Since $x \in W, x_{1}, \ldots, x_{j} \in W$. Hence $x R^{j} y$. In an analogous way it can be seen that the subframe generated by $u$ is $n$-symmetric.

Theorem 4.9 The normal modal logic of the class of frames that satisfy the condition (*) above is precisely the logic Kn.4B.

Proof: Suppose that $\varphi$ is not a theorem of Kn.4B. Let $u$ be a possible world in the canonical model for Kn.4B in which $\varphi$ is false. Take the submodel generated by $u$. By the last lemma the frame of this model is weakly $n$-transitive and $n$-symmetric. Hence by Lemma 4.7 it satisfies the condition. By the theorem on generated subframes we have that $\varphi$ is not valid in a frame that satisfies the condition. Hence using Lemma 4.6 we have the theorem.

In the next part of this section we study the relations that hold between the logics Kn.4B, Kn+1.4B and K4B. First of all we prove a lemma.

Lemma 4.10 Every weakly $n$-transitive and $n$-symmetric frame is a weakly $n+1$ transitive and $n+1$-symmetric frame.

Proof: Suppose that $\langle W, R\rangle$ is a weakly $n$-transitive and $n$-symmetric frame. We know that it is weakly $n+1$-transitive. To see that it is $n+1$-symmetric suppose that there is $j \leq n+1$ such that $x R^{j} y$. If $j<n+1$, by $n$-symmetry there is $j^{\prime} \leq n$ such that $y R^{j^{\prime}} x$. If $j=n+1$, by weak $n$-transitivity there is $j^{\prime} \leq n$ such that $x R^{j^{\prime}} y$. Therefore by $n$-symmetry there is $j^{\prime \prime} \leq n$ such that $y R^{j^{\prime \prime}} x$.

\section{Proposition 4.11 For every $n>0, \mathbf{K n}+\mathbf{1 . 4 B} \varsubsetneqq$ Kn.4B.}

Proof: The frame used in the proof of Proposition 3.11 is weakly $n+1$-transitive and $n+1$-symmetric, but it is not weakly $n$-transitive. Hence $[n] p \rightarrow \square^{n+1} p$ is not valid in it. Therefore this formula is not a theorem of $\mathbf{K n + 1 . 4 B}$. On the other hand, as we already know, the axiom of $\mathbf{K n + 1 . 4}$ is a theorem of $\mathbf{K n . 4 B}$. Also by Lemma 4.10 we know that every weakly $n$-transitive and $n$-symmetric frame is weakly $n+1$ transitive and $n+1$-symmetric. Since the logics we consider are complete relative to their classes of frames we obtain the proposition. 


\section{Proposition 4.12 K1.4B $\varsubsetneqq$ K4B.}

Proof: It is evident that $\varphi \rightarrow \square(\diamond \varphi \vee \varphi)$ is a theorem of K4B. And we know that K1.4 is included in K4. On the other hand the frame used in the proof of Proposition 3.12 is symmetric and weakly $n$-transitive but it is not transitive. Hence the inclusion is proper since these logics are complete relatively to their classes of frames.

\section{Proposition 4.13}

(1) If $n \neq 0$, Kn.4 $\mp \mathbf{K n . 4 B . ~}$

(2) $\mathrm{KO} .4=\mathrm{K0.4B}$.

(3) $\mathrm{K4B} \subsetneq$ K0.4.

Proof: (1) Obviously the inclusion holds. To see that it is proper let us consider the frame with domain $\{0, \ldots, n\}$ and relation the defined by

$$
i R j \text { iff } j=i+1 \text {. }
$$

This relation is not $n$-symmetric since $0 R 1$ but for every $j \leq n$ it is not the case that $1 R^{j} 0$. Obviously the frame is weakly $n$-transitive. Since the logics are complete relative to their classes of frames we get the desired result.

(2) is clear since the axiom $\mathbf{0 B}$ is $\varphi \rightarrow \varphi$.

(3) The K0.4 frames are the ones whose relation is included in the identity. So they are transitive and symmetric. On the other hand there are obviously transitive and symmetric frames whose relation is not included in the identity. Since both logics are complete relative to their classes of frames we have the desired result.

We do not know if the infimum in the lattice of normal modal logics of the collection of logics $\mathbf{K n . 4 B}$ is the logic $\mathrm{K}$, but our conjecture is that it is not.

The relationships between the logics we have been studying are summarized in the next diagram:

$\ldots q \mathrm{Kn}+1.4 \mp \mathrm{Kn} .4 \mp \ldots q \mathrm{~K} \mathbf{1 . 4} \varsubsetneqq \mathrm{K} 4 \mp \mathrm{K0.4}$

$\mathbf{K}$

$$
\ldots \varsubsetneqq K n+1.4 B \varsubsetneqq K n .4 B \varsubsetneqq \ldots \varsubsetneqq K 1.4 B \varsubsetneqq K 4 B \varsubsetneqq K 0.4 B
$$

To conclude this section the next Proposition states via translations the relation between the logics Kn.4B and S5.

Proposition 4.14 For every $n \geq 1$ and every modal formula $\varphi, \varphi$ is a theorem of S5 if and only if $\varphi^{(n)}$ is a theorem of Kn.4B.

Proof: $(\Rightarrow)$ If $\varphi^{(n)}$ is not a theorem of $\mathbf{K n . 4 B}$, let $\langle W, R\rangle$ be an $n$-transitive and $n$ symmetric frame where $\varphi^{(n)}$ is not valid. By Lemma 3.15, $\varphi$ is not valid in the frame $\langle W, R \mathrm{n}\rangle$. But by Lemma 3.16 and the observation made after Definition 4.2, this frame is transitive, reflexive and symmetric, and therefore is a frame for S5. Hence $\varphi$ is not a theorem of $\mathbf{S 5}$.

$(\Leftarrow)$ If $\varphi$ is not a theorem of $\mathbf{S 5}$, let $\langle W, R\rangle$ be a transitive, reflexive and symmetric frame in which $\varphi$ is not valid. It exists since $\mathbf{S 5}$ is complete relative to the class of these frames. Since $R \mathrm{n}$ is $R$, by Lemma $4.8 \varphi^{(n)}$ is not valid in the frame $\langle W, R\rangle$. Therefore $\varphi^{(n)}$ is not valid in a weakly $n$-transitive and $n$-symmetric frame. Hence it is not a theorem of $\mathbf{K n . 4 B}$. 
5 The finite model property In this last section we show that the logics Kn.4B have the finite model property and hence the finite frame property.

Theorem 5.1 The logics Kn.4B have the finite model property, and therefore are decidable.

Proof: Consider the canonical model for Kn.4B. Let $\varphi$ be a formula that is not a theorem of Kn.4B. Let $u$ be a possible world in the canonical model on which $\varphi$ is false. Take the submodel generated by $u,\langle W, R, e\rangle$. By Lemma 4.8 it is a model with a weakly $n$-transitive and $n$-symmetric frame. By Lemma 4.7 it also satisfies the condition

$$
\forall x y \in W\left(x \neq y \rightarrow \exists j \leq n x R^{j} y\right) .
$$

Consider now the filtration of this model by the finite set of subformulas of $\varphi$ with the greatest appropriate relation $R^{\prime}\left([x] R^{\prime}[y]\right.$ if and only if there is $w \in[x]$ and there is $v \in[y]$ such that $w R v$ ). This model is finite. By the theorem on filtrations $\varphi$ is false in $[u]$. Let us see that the frame of the filtration we have taken is weakly $n$-transitive and $n$-symmetric. First we prove that it is weakly $n$-transitive. If $[x] R^{\prime n+1}[y]$ let $w \in[x]$ and $v \in[y]$ be such that $w R v$. If $w=v,[x]=[y]$. If $w \neq v$, let $j \leq n$ be such that $w R^{j} v$. Let $w, x_{1}, \ldots, x_{j}, v$ be an $R$-sequence. Then $[w],\left[x_{1}\right], \ldots,\left[x_{j}\right],[v]$ is an $R^{\prime}$-sequence. Hence, $[x] R^{\prime j}[y]$. To conclude we see that it is $n$-symmetric. If $[x] R^{\prime j}[y]$ for some $j \leq n$ and $j=0,[x]=[y]$ and $[y] R^{\prime j}[x]$. If $j \neq 0$, take $w \in[x]$ and $v \in[y]$. If $w=v,[x]=[y]$ and $[y] R^{\prime 0}[x]$. If $w \neq v$ let $j^{\prime} \leq n$ be such that $w R^{j^{\prime}} v$. By $n$-symmetry let $j^{\prime \prime} \leq n$ be such that $v R^{j^{\prime \prime}} w$. Reasoning as in the case of the weak $n$-transitivity we have that $[y] R^{\prime} j^{\prime \prime}[x]$. Therefore $\varphi$ is not valid in a model on a finite weakly $n$-transitive and $n$-symmetric frame, a finite model of Kn.4B.

Acknowledgment This work is partially supported by grant PB90-0465-C02-01 of the Spanish DGICYT. The author wishes to thank Pilar Dellunde and J. M. Font for their comments on a previous version of this paper.

\section{REFERENCES}

[1] Blok, W. and P. Köhler, "Algebraic semantics for quasi-classical modal logics," The Journal of Symbolic Logic, vol. 48 (1983), pp. 941-964.

[2] Goldblatt, R., "Arithmetical necessity, provability and intuitionistic logic," Theoria, vol. 44 (1978), pp. 38-46.

[3] Hughes G. E. and M. J. Cresswell, A Companion to Modal Logic, Methuen, London, 1984.

[4] Rautenberg, W., Klassische und nichtklassische Aussagenlogik, Vieweg und Sohn, Braunschweig, 1979.

[5] Segerberg, K., “A note on the logic of elsewhere," Theoria, vol. 46 (1980), pp. 183-187.

[6] von Wright, G. H., "A modal logic of place," pp. 65-73 in The Philosophy of Nicholas Rescher, edited by E. Sosa, Reidel, Dordrecht, 1979. 
Departament de Lògica, Història i Filosofia de la Ciència Universitat de Barcelona,

08071 Barcelona, Spain

email: jansana@cerber.ub.es 